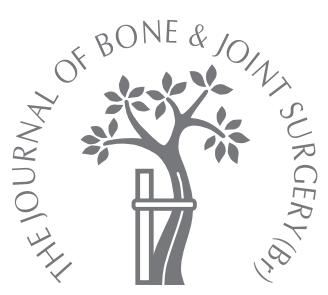

B. S. Kim, M. Knupp,

L. Zwicky,

J. W. Lee,

B. Hintermann

From Kantonsspital, Liestal, Switzerland

B. S. Kim, MD, Orthopaedic Surgeon

Department of Orthopaedic

Surgery

Inha University Hospital, 7-206,

$3 \mathrm{Ga}$, Sinheung-dong, Jung-gu

Incheon, 400-711 Republic of

Korea.

M. Knupp, MD, Orthopaedic Surgeon

L. Zwicky, MS, Research

Assistant

a. Hintermann, MD,

Professor

Clinic of Orthopaedic Surgery

Kantonsspital, Rheinstrasse 26

$\mathrm{CH}-4410$, Liestal, Switzerland.

드 J.W. Lee, MD, PhD, Professor Department of Orthopaedic

Surgery

Yonsei University College of

Medicine, Seoul, Republic of

Korea.

Correspondence should be sent to Professor B. Hintermann;

e-mail:beat.hintermann@ksli.ch

(c)2010 British Editorial Society

of Bone and Joint Surgery

doi:10.1302/0301-620X.92B11.

$24452 \$ 2.00$

$J$ Bone Joint Surg $[\mathrm{Br}]$

2010;92-B:1540-7.

Received 14 January 2010;

Accepted after revision 12 July

2010

\title{
Total ankle replacement in association with hindfoot fusion
}

\author{
OUTCOME AND COMPLICATIONS
}

We report the clinical and radiological outcome of total ankle replacement performed in conjunction with hindfoot fusion or in isolation. Between May 2003 and June 2008 , 60 ankles were treated with total ankle replacement with either subtalar or triple fusion, and the results were compared with a control group of 288 ankles treated with total ankle replacement alone.

After the mean follow-up of $\mathbf{3 9 . 5}$ months (12 to 73), the ankles with hindfoot fusion showed significant improvement in the mean visual analogue score for pain $(p<0.001)$, the mean American Orthopaedic Foot and Ankle Society score $(p<0.001)$, and the mean of a modified version of this score $(p<0.001)$. The mean visual analogue pain score $(p=0.304)$ and mean modified American Orthopaedic Foot and Ankle Society score $(p=0.119)$ were not significantly different between the hindfoot fusion and the control groups. However, the hindfoot fusion group had a significantly lower mean range of movement $(p=0.009)$ and a higher rate of posterior focal osteolysis $(p=0.04)$. Both groups showed various complications $(p=0.131)$ and failure occurring at a similar rate $(p=0.685)$.

Subtalar or triple fusion is feasible and has minimal adverse effects on ankles treated with total ankle replacement up to midterm follow-up. The clinical outcome of total ankle replacement when combined with hindfoot fusion is comparable to that of ankle replacement alone. Thus, hindfoot fusion should be performed in conjunction with total ankle replacement when indicated.

Malalignment is a major risk factor for early failure in total ankle replacement (TAR), and most authors agree that it needs to be addressed before or simultaneously with any joint replacement. ${ }^{1-7}$ Furthermore, patients with osteoarthritis of the ankle joint frequently have degenerative disease affecting the neighbouring joints. ${ }^{8-10}$ For these reasons, additional procedures are commonly performed, with several papers describing the combination of TAR and subtalar or triple fusion. ${ }^{4,11,12}$

Subtalar joint or triple fusion are used to correct deformity, relieve pain, achieve a plantigrade foot, and give good results. ${ }^{13-18}$ However, problems arising in adjacent joints are of concern since the loss of mechanism to dissipate forces in the subtalar and midtarsal regions places abnormal stress on the ankle and leads to increased wear or degeneration. ${ }^{14,17-21}$

If a TAR is implanted in the presence of a fused hindfoot, the prosthesis is theoretically exposed to higher loads. However, how a fused hindfoot affects a TAR and the clinical outcome are poorly understood. Additionally there are concerns that if the vascularity of the talus is adversely affected, there could be delayed healing and the tolerance for malpositioned components might be affected. Finally, consideration should be given to whether such intervention should be simultaneous or staged, and if isolated, whether subtalar, double or triple fusion should be performed. This study aimed to address the effect of hindfoot fusion on TAR by comparing clinical and radiological outcomes of TARs performed with and without hindfoot fusion.

\section{Patients and Methods}

Between May 2003 and June 2008, 362 primary TARs were performed on 347 patients, including 15 patients who underwent bilateral procedures. The indication for surgery was severe pain in an arthritic ankle joint that was refractory to non-operative treatment. The Hintegra (Newdeal SA, Lyon, France) third generation total ankle system was used in all cases. We only included primary replacements, excluding revision procedures and conversion of ankle arthrodesis to TAR. In order to 
Table I. Demographic data of the two groups

\begin{tabular}{|c|c|c|c|}
\hline & Hindfoot fusion group & Control group & \\
\hline & ( $n=60$ ankles) & ( $n=288$ ankles) & p-value \\
\hline Mean (SD) age in years & $61.6(13.6)$ & $61.5(12.3)$ & \\
\hline Male:female ratio & 23:37 & $162: 126$ & $<0.05^{\dagger}$ \\
\hline Right:left ratio & $31: 29$ & $152: 136$ & \\
\hline Mean (SD) weight in kg & $75.7 \quad(19.2)$ & $80.6 \quad(14.9)$ & $<0.05^{\ddagger}$ \\
\hline Mean (SD) height in $\mathrm{cm}$ & $168.2(8.6)$ & $171.6(9.0)$ & $<0.05^{\ddagger}$ \\
\hline Mean (SD) body mass index in $\mathrm{kg} / \mathrm{m}^{2}$ & $26.7(6.0)$ & $27.3 \quad(4.1)$ & \\
\hline Mean (SD) follow-up in months & $36.9(16.9)$ & $40.1 \quad(18.1)$ & \\
\hline \multicolumn{4}{|l|}{ Pre-operative diagnosis (\%) } \\
\hline Degenerative OA* & $4(6.7)$ & $16(5.6)$ & $<0.005^{\dagger}$ \\
\hline Post-traumatic OA & $43(71.7)$ & $253(87.8)$ & \\
\hline Rheumatoid arthritis & $8(13.3)$ & $6(2.1)$ & \\
\hline Other & $5(8.3)$ & $13(4.5)$ & \\
\hline \multicolumn{4}{|l|}{ Physical stress at job $(\%)^{\pi}$} \\
\hline None & $4(6.7)$ & $4(1.4)$ & \\
\hline Mild & $21(35)$ & $125(43.4)$ & \\
\hline Moderate & $26(43.3)$ & $109(37.8)$ & \\
\hline Hard & $9(15)$ & $50(17.4)$ & \\
\hline \multicolumn{4}{|l|}{ Quality of life before operation (\%) } \\
\hline Good & $11(18.3)$ & $78(27.1)$ & \\
\hline Moderate & $22(36.7)$ & $114(39.6)$ & \\
\hline Bad & $27(45)$ & $96(33.3)$ & \\
\hline \multicolumn{4}{|l|}{ * OA, osteoarthritis } \\
\hline \multicolumn{4}{|l|}{ † chi-squared test or Fisher's exact test } \\
\hline \multicolumn{4}{|l|}{ ‡ two-sample $t$-test, $\mathrm{p}<0.05$} \\
\hline I quantified with an institutional qu & & & \\
\hline
\end{tabular}

evaluate the effect of hindfoot fusion, patients who had previously undergone midfoot or forefoot fusion were also excluded. A total of 13 patients (14 ankles) were lost to follow-up: seven patients died, four were from or moved abroad, and two declined to attend for review. The remaining 334 patients ( 348 TARs) were the basis for the clinical and radiological evaluation of this study, with a mean follow-up of 39.5 months (12 to 73 ).

These patients were separated into two groups depending on their hindfoot status: hindfoot fusion (TAR with hindfoot fusion; 60 ankles) and a control group (isolated TAR; 288 ankles). Clinical and radiological outcomes, complications and failure rates were compared between the groups. Revision or conversion to arthrodesis was regarded as a failure. The demographic data of the two groups are shown in Table I.

In the hindfoot fusion group, $28(46.7 \%)$ had TAR with isolated subtalar fusion, $29(48.3 \%)$ with subtalar and talonavicular (double) arthrodesis, and three $(5.0 \%)$ with subtalar, talonavicular and calcaneocuboid (triple) arthrodesis. Hindfoot fusion was performed simultaneously with TAR in 39 ankles $(65.0 \%)$, while $17(28.3 \%)$ had their hindfoot fused at a mean of 8 years $(0.25$ to 38$)$ before TAR, and four $(6.7 \%)$ had hindfoot fusion at a mean of 14.3 months ( 8 to 23 ) after TAR. The indications for hindfoot fusion were correction of deformity in 23 ankles
(38.3\%, 14 varus and nine valgus malalignment), posttraumatic osteoarthritis in $20(33.3 \%)$, primary osteoarthritis in eight $(13.3 \%)$, and other causes in nine $(15 \%$, six rheumatoid arthritis, two gouty arthropathy, one haemophilia).

Additional surgeries. TAR was performed using a standard surgical technique, ${ }^{10,22}$ and in the presence of malalignment or ligamentous instability, appropriate additional procedures were conducted before or together with TAR as shown in Table II.

Clinical evaluation. All patients were evaluated preoperatively and at two weeks, six weeks, three months, six months and one year post-operatively and annually thereafter. Clinical evaluations were performed by a research assistant (LZ) who was not involved in the surgery.

Pain was rated using the visual analogue scale (VAS) ranging from 0 (no pain) to 10 (maximum pain). The range of movement (ROM) of the ankle-hindfoot complex was measured using a goniometer along the lateral border of the leg and foot. The American Orthopaedic Foot and Ankle Society (AOFAS) ankle and hindfoot scoring system ${ }^{23}$ was used to evaluate the function. We also modified this score ${ }^{19}$ by excluding the hindfoot movement (six points) since this is always eliminated after hindfoot fusion. The modified AOFAS score was thus composed of 94 points, allowing us to compare the function status of the two groups without the 
Table II. Associated procedures performed together with primary total ankle replacement

\begin{tabular}{|c|c|c|c|}
\hline & Hindfoot fusion group & Control group & p-value* \\
\hline Osteotomies (\%) & $10(16.7)$ & $24(8.3)$ & 0.057 \\
\hline Calcaneal osteotomy & 7 & 9 & \\
\hline Supramalleolar osteotomy & 0 & 6 & \\
\hline Medial malleolar osteotomy & 0 & 1 & \\
\hline Metatarsal osteotomy & 1 & 4 & \\
\hline Cuneiform osteotomy & 2 & 2 & \\
\hline Tibial osteotomy & 0 & 1 & \\
\hline Fibular osteotomy & 0 & 1 & \\
\hline Ligament/tendon procedures (\%) & $14(23.3)$ & $53(18.4)$ & 0.372 \\
\hline Medial augmentation & 0 & 1 & \\
\hline Medial and lateral augmentation & 1 & 1 & \\
\hline Peroneus longus transfer & 2 & 6 & \\
\hline Posterior tibialis transfer & 0 & 2 & \\
\hline Flexor digitorum longus transfer & 0 & 1 & \\
\hline Flexor hallucis longus transfer & 0 & 1 & \\
\hline Tendo Achillis lengthening & 11 & 42 & \\
\hline
\end{tabular}

* chi-squared test or Fisher's exact test

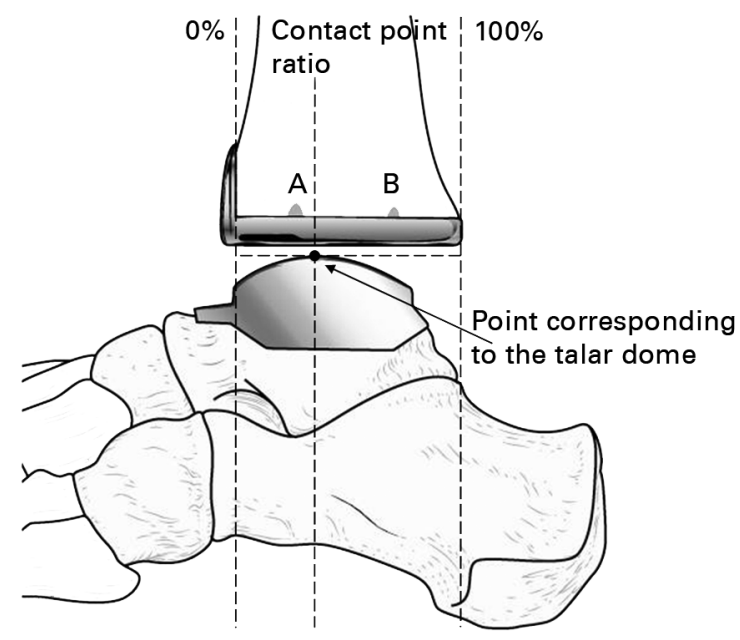

Fig. 1

Diagram showing contact point ratio with the foot in neutral position. The apex of the talar component or the point corresponding to the talar dome indicates the central area of contact with the tibial component. The contact point ratio is the ratio of the contact point of the talar component $(A)$ to the length of the tibial loading plate $(A+B)$. The desired contact point is between $40 \%$ and $45 \%$.

confounding variable of hindfoot fusion. Patients were also asked to indicate their quality of life as either good, moderate or bad, state their satisfaction with the procedure on a categorical scale of very satisfied, satisfied, satisfied with reservations and dissatisfied, and whether he/she would receive the same operation again in a similar situation.

Radiological evaluation. Weight-bearing true anteroposterior (AP) and lateral views were obtained fluoroscopically at each visit. Serial radiographs were evaluated to determine the position of the components, presence of any loosening defined as a change in position $>2^{\circ}$ and for a progressive radiolucency of $>2 \mathrm{~mm}$ in either view, ${ }^{4}$ heterotopic ossification and osteolysis. Angular and linear values were measured using Image Access special metric software (PIC Systems AG, Glattbrugg, Switzerland). In order to evaluate the sagittal balance of the replaced ankle and the relocation of the talus within the mortise, we measured the contact point ratio ${ }^{10}$ on standing radiographs immediately after surgery and at the last follow-up. This is a ratio of the contact point of the talar component to the length of the tibial loading plate; the optimal contact point ratio is between $40 \%$ and $45 \%$ (Fig. 1). ${ }^{10}$ The pre-operative varus or valgus angulation of the ankle was measured between the anatomical axis of the tibia and a line perpendicular to the articular surface of the talus. ${ }^{5} \mathrm{Mal}-$ position of the tibial component was defined as the placement of the component with an angular deviation $>5^{\circ}$ to the longitudinal tibial axis. ${ }^{22}$

Statistical analysis. Statistical data were analysed by using SPSS version 15.0 software (SPSS Inc., Chicago, Illinois). A paired $t$-test was used to evaluate the clinical changes before and after surgery. For a comparison of the outcomes between the two groups, a two-sample $t$-test for continuous variables and a chi-squared (or Fisher's exact, as appropriate) test for nominal data were used. A p-value $<0.05$ was considered significant.

\section{Results}

Pain in the ankle in the hindfoot fusion group was significantly reduced $(\mathrm{p}<0.001)$, and there was no difference in the mean VAS compared to the control group, both pre-operatively ( $\mathrm{p}=$ $0.587)$ and at final follow-up ( $p=0.304)$ (Table III).

Ankles in the hindfoot fusion group showed significant improvement in both the AOFAS $(\mathrm{p}<0.001)$ and the modified AOFAS score $(\mathrm{p}<0.001)$ (Table III). When compared with the control group, the AOFAS score of the hindfoot fusion group was lower before the operation $(\mathrm{p}<0.001)$ 
Table III. Clinical assessment (mean, SD) of the two groups

\begin{tabular}{|c|c|c|c|}
\hline & Hindfoot fusion group & Control group & p-value \\
\hline \multicolumn{4}{|l|}{ Visual analogue scale } \\
\hline Pre-operative & $6.8(2.0)$ & $6.7(1.8)$ & $0.587^{\dagger}$ \\
\hline Last follow-up & $2.5(2.1)$ & $2.8(2.4)$ & $0.304^{\dagger}$ \\
\hline Improvement & $4.4(2.6)$ & $4.0(3.0)$ & $0.351^{\dagger}$ \\
\hline p-value & $<0.0001^{\ddagger}$ & $<0.0001^{\ddagger}$ & \\
\hline \multicolumn{4}{|l|}{ AOFAS $^{*}$ score } \\
\hline Pre-operative & $35.5(16.4)$ & $44.4(16.5)$ & $<0.0001^{\dagger}$ \\
\hline Last follow-up & $66.6(17.7)$ & $73.4(18.2)$ & $0.011^{\dagger}$ \\
\hline Improvement & $31.1(22.6)$ & $30.2(23.1)$ & $0.788^{\dagger}$ \\
\hline$p$-value ${ }^{\ddagger}$ & $<0.0001^{\ddagger}$ & $<0.0001^{\ddagger}$ & \\
\hline \multicolumn{4}{|l|}{ Modified AOFAS score } \\
\hline Pre-operative & $32.0(16.6)$ & $41.4(16.7)$ & $<0.0001^{\dagger}$ \\
\hline Last follow-up & $66.6(17.7)$ & 70.7 (17.7) & $0.119^{\dagger}$ \\
\hline Improvement & $34.7(22.7)$ & $30.3(22.9)$ & $0.205^{\dagger}$ \\
\hline $\mathrm{p}$-value & $<0.0001^{\ddagger}$ & $<0.0001^{\ddagger}$ & \\
\hline \multicolumn{4}{|l|}{ Range of movement } \\
\hline Pre-operative & $25.0(12.8)$ & $30.14(15.3)$ & $0.007^{\dagger}$ \\
\hline Last follow-up & $29.5(10.6)$ & $34.3(10.6)$ & $0.009^{\dagger}$ \\
\hline Improvement & $4.1(17.7)$ & 3.4 (14.9) & $0.786^{\dagger}$ \\
\hline $\mathrm{p}$-value & $0.141^{\ddagger}$ & $0.001^{\ddagger}$ & \\
\hline Satisfaction (\%) & & & $0.707^{\S}$ \\
\hline Very satisfied & $25(41.7)$ & $105(36.5)$ & \\
\hline Satisfied & $21(35.0)$ & $120(41.7)$ & \\
\hline Satisfied with reservation & $11(18.3)$ & $41(14.2)$ & \\
\hline Dissatisfied & $3(5.0)$ & $22(7.6)$ & \\
\hline Quality of life (\%) & & & $0.303^{\S}$ \\
\hline Good & $32(53.3)$ & $185(64.2)$ & \\
\hline Moderate & $21(35.0)$ & 79 (27.4) & \\
\hline Bad & $7(11.7)$ & $24(8.3)$ & \\
\hline Repetition of operation (\%) & & & $0.780^{\S}$ \\
\hline Yes & $55(91.7)$ & $267(92.7)$ & \\
\hline No & $5(8.3)$ & $21(7.3)$ & \\
\hline
\end{tabular}

and at the final follow-up ( $\mathrm{p}=0.011)$. However, the modified AOFAS score showed no significant difference with the control group at final follow-up $(\mathrm{p}=0.119)$.

The ROM of the ankle of the hindfoot fusion group was significantly less pre-operatively $(\mathrm{p}=0.007)$ and post-operatively $(\mathrm{p}=0.009)$ compared to the control group, and was not significantly improved after the operation $(\mathrm{p}=0.141)$ (Table III). While the ROM of the control group was significantly improved $(\mathrm{p}<0.001)$, the mean amount of gained movement of $3.4^{\circ}$ (SD 14.9) was similar to the mean gain in the hindfoot fusion group of $4.1^{\circ}$ (SD 17.7; $\mathrm{p}=0.786$ ).

The two groups showed a similar distribution of level of satisfaction ( $\mathrm{p}=0.707)$, with less than $10 \%$ of dissatisfied patients in each group. Likewise, a similar proportion of patients in the hindfoot fusion $(88.3 \%)$ and control groups
$(91.6 \%)$ rated their quality of life as good or moderate $(\mathrm{p}=0.303)$ (Table III). Over $90 \%$ of patients in each group would undergo the same operation again.

The hindfoot fusion group was further analysed to compare the clinical outcomes of isolated subtalar fusion $(\mathrm{n}=28)$ with double or triple fusion $(\mathrm{n}=32)$. No differences were observed between the two subgroups in all clinical measures, including post-operative VAS $(\mathrm{p}=0.262)$, AOFAS score $(\mathrm{p}=0.359)$, modified AOFAS score $(\mathrm{p}=0.359)$, and range of ankle movement $(\mathrm{p}=0.983)$.

Radiologically, focal osteolysis around the posterior tip of the tibial loading plate occurred more often in the hindfoot fusion group than the control group $(\mathrm{p}=0.04)$ (Table IV). There was also a tendency towards more bony overgrowth around components in the hindfoot fusion group, 
Table IV. Details (mean, SD) of the radiological findings of the two groups

\begin{tabular}{llll}
\hline & Hindfoot fusion group & Control group & p-value \\
\hline$\alpha$ angle $\left(^{\circ}\right)$ & $88.6(2.7)$ & $87.8(7.9)$ & $0.456^{*}$ \\
$\beta$ angle $\left(^{\circ}\right)$ & $87.4(3.4)$ & $87.2(6.0)$ & $0.874^{*}$ \\
Focal radiolucency (\%) & $13(24.1)$ & $35(12.8)$ & $0.055^{\dagger}$ \\
Posterior focal osteolysis (\%) & $11(18.3)$ & $26(9)$ & $0.040^{\dagger}$ \\
Heterotopic ossification (\%) & $8(14.8)$ & $38(13.9)$ & $0.832^{\dagger}$ \\
Bony overgrowth around component (\%) $22(40.7)$ & $76(27.7)$ & $0.073^{\dagger}$
\end{tabular}

* two-sample $t$-test

$\dagger$ chi-squared test or Fisher's exact test

Table V. Relocation of talus; by contact point ratio (mean, SD)

\begin{tabular}{|c|c|c|c|}
\hline & Hindfoot fusion group & Control group & p-value ${ }^{*}$ \\
\hline \multicolumn{4}{|l|}{ All $(n=348)$} \\
\hline Post-operative & $38.8(6.9)$ & $42.2(6.4)$ & 0.002 \\
\hline Last follow-up & $41.3(5.6)$ & $43.4(6.5)$ & 0.051 \\
\hline p-value ${ }^{\dagger}$ & 0.002 & 0.137 & \\
\hline \multicolumn{4}{|c|}{ Anterior facing (posterior slope angle ${ }^{\ddagger}<86^{\circ}, \mathrm{n}=106$ ) } \\
\hline Post-operative & $37.3(7.0)$ & $42.7(5.9)$ & 0.003 \\
\hline Last follow-up & $38.6(4.9)$ & $44.3(6.1)$ & 0.001 \\
\hline p-value ${ }^{\dagger}$ & 0.399 & 0.076 & \\
\hline \multicolumn{4}{|c|}{ Neutral (posterior slope angle: $86^{\circ}$ to $90^{\circ}, \mathrm{n}=191$ ) } \\
\hline Post-operative & $40.3(6.5)$ & $43.1(6.1)$ & 0.087 \\
\hline Last follow-up & $42.8(5.6)$ & $43.8(7.2)$ & 0.549 \\
\hline $\mathrm{p}_{\text {-value }}^{\dagger}$ & 0.027 & 0.707 & \\
\hline \multicolumn{4}{|c|}{ Posterior facing (posterior slope angle $>90^{\circ}, \mathrm{n}=51$ ) } \\
\hline Post-operative & $37.3(7.4)$ & $40.2(7.3)$ & 0.301 \\
\hline Last follow-up & $42.6(5.4)$ & $41.3(5.9)$ & 0.575 \\
\hline $\mathrm{p}$-value ${ }^{\dagger}$ & 0.009 & 0.301 & \\
\hline
\end{tabular}

but this did not reach statistical significance $(p=0.073)$. Heterotopic ossification was observed in both groups with a similar incidence $(\mathrm{p}=0.832)$.

Three ankles $(5 \%)$ in the hindfoot fusion group and ten ankles $(3.5 \%)$ in the control group had pre-operative varus or valgus of the ankle of $>15^{\circ}$. The pre-operative coronal deformity at the ankle joint did not have an association with the clinical outcome within the hindfoot fusion group (postoperative mean VAS $(\mathrm{p}=0.510)$, the mean AOFAS score $(\mathrm{p}=0.471)$, the mean modified AOFAS score $(\mathrm{p}=0.471)$, mean range of ankle movement $(\mathrm{p}=0.370)$ ) and the control group (post-operative mean VAS $(\mathrm{p}=0.967)$, mean AOFAS score $(p=0.895)$, mean modified AOFAS score $(p=0.876)$, mean range of ankle movement $(\mathrm{p}=0.857))$.

Four ankles $(6.7 \%)$ in the hindfoot fusion group and 22 ankles $(7.6 \%)$ in the control group had a malpositioned tibial component. We compared the malpositioned ankles, and found no difference in the mean post-operative VAS of 1.25 (SD 1.89) in the hindfoot fusion group $v s$ a mean of 3.05 (SD 2.19) in the control group $(\mathrm{p}=0.139)$. Similarly the mean AOFAS scores of 70.5 (SD 13.0) vs 72.7 (SD 20.7) for the two groups respectively were not significantly different $(\mathrm{p}=0.839)$, and likewise the mean modified AOFAS scores of 70.5 (SD 13.0) vs 69.5 (SD 19.6), respectively, were not significantly different $(\mathrm{p}=0.920)$. Four malpositioned ankles in the control group had focal osteolysis around the tibial component, but none of them progressed to failure.

The talus or the hindfoot complex was anteriorly subluxated in the hindfoot fusion group with a mean contact point ratio of $38.8 \%$ (SD 6.9) in the first post-operative standing lateral radiograph (Table V). However, the hindfoot complex was relocated within the mortise with a mean contact point ratio of $41.3 \%$ (SD 5.6) at final follow-up. Such relocation of the talus was only observed in ankles in the hindfoot fusion group, and especially in those with the posterior slope angle $>86^{\circ} .22$

Complications and revision operations. Various complications developed in both groups, but there was no significant difference in the complication rates $(\mathrm{p}=0.131)$ (Table VI). 
Table VI. Complications and failures

\begin{tabular}{llll}
\hline & Hindfoot fusion group & Control group & p-value $^{*}$ \\
\hline Overall complications (ankles, \%) & $14(23.3)$ & $44(15.3)$ & 0.131 \\
Painful arthrofibrosis (\%) & $6(10)$ & $16(5.6)$ & 0.239 \\
Bony impingement (\%) & $3(5)$ & $13(4.5)$ & 0.745 \\
Component loosening (\%) & $3(5)$ & $7(2.4)$ & 0.386 \\
Instability/polyethylene dislocation (\%) & $3(5)$ & $3(1)$ & 0.066 \\
Osteolytic cyst formation (\%) & $1(1.7)$ & $2(0.7)$ & 0.434 \\
Malleolar fatigue fracture (\%) & $0(0)$ & $2(0.7)$ & 1.0 \\
Wound healing problems (\%) & $0(0)$ & $2(0.7)$ & 1.0 \\
Intra-articular infection (\%) & $2(3.3)$ & $1(0.3)$ & 0.078 \\
Failure (\%) & $2(3.3)$ & $8(2.8)$ & 0.685 \\
Component revision & 1 & 4 & \\
Ankle arthrodesis & 1 & 4 & \\
\hline
\end{tabular}

* chi-squared test or Fisher's exact test

Table VII. Complications and failures by the timing of hindfoot fusion

\begin{tabular}{|c|c|c|c|}
\hline & \multicolumn{2}{|l|}{ Hindfoot fusion group } & \multirow[b]{2}{*}{ p-value ${ }^{*}$} \\
\hline & Simultaneous fusion $(n=39)$ & Two-stage fusion $(n=21)$ & \\
\hline Overall complications (ankles, \%) & $6(15.4)$ & $8(38.1)$ & 0.061 \\
\hline Prosthesis-related problems & $4(10.3)$ & $3(14.3)$ & 0.687 \\
\hline Instability/polyethylene dislocation & $3(7.7)$ & $0(0.0)$ & \\
\hline Component loosening & $1(2.6)$ & $2(9.5)$ & \\
\hline Osteolysis & $0(0)$ & $1(4.8)$ & \\
\hline Soft tissue-related or secondary problems & $3(7.7)$ & $6(28.6)$ & 0.054 \\
\hline Scarring/arthrofibrosis & $2(5.1)$ & $4(19.0)$ & \\
\hline Bony impingement & $1(2.6)$ & $2(9.5)$ & \\
\hline Intra-articular infection & $1(2.6)$ & $1(4.8)$ & \\
\hline Wound healing problems & $0(0)$ & $0(0)$ & \\
\hline Failure (\%) & $1(2.6)$ & $1(4.8)$ & 1.0 \\
\hline
\end{tabular}

* chi-squared test (Fisher's exact test)

We also evaluated the complication rates depending on the timing of hindfoot fusion, whether performed simultaneously with TAR or separately. There was a tendency towards more frequent development of soft-tissue related or secondary problems including scarring and bony impingement in the two-stage fusion group $(\mathrm{p}=0.054)$, whereas instability or dislocation of the polyethylene component occurred only in the simultaneous fusion group (Table VII).

No significant difference was observed in the cumulative failure rate between the two groups $(\mathrm{p}=0.685)$ (Table VI). A total of two ankles $(3.3 \%)$ in the hindfoot fusion group and eight ankles $(2.8 \%)$ in the control group underwent revision (Table VIII). The reason for failure was aseptic loosening in seven ankles, painful arthrofibrosis in two ankles and dislocation of the polyethylene component in one. In five TARs the revision was managed by changing the metallic component, while the other five were converted to an arthrodesis.

\section{Discussion}

This study presents, to the best of our knowledge, the first clinical report of TAR performed in conjunction with fusion of the hindfoot. The outcomes in the hindfoot fusion group were generally satisfactory and no worse than those in the control group. Significant improvements were observed in most clinical parameters in both groups, including VAS, AOFAS score and modified AOFAS score. The modified AOFAS score was significantly lower in the hindfoot fusion group before the operation but improved to a degree comparable to the control group at final followup. This revealed that other than hindfoot inversion and eversion, the function of the replaced ankle and the hindfoot complex in the hindfoot fusion group was equivalent to that of the control group. The two groups showed no difference in terms of satisfaction, self-rated quality of life and willingness to undergo the same operation again. The complication rate and the cumulative failure rate in the hindfoot fusion group were not higher than those of the control group. However, a high rate of posterior focal osteolysis was observed in the hindfoot fusion group, suggesting accelerated wear of polyethylene which might prejudice the long-term outcome. Whether this finding is the result of the loss of a compensatory function of the subtalar joint ${ }^{24}$ such that a slight degree of malalignment can lead to the eccentric transmission of force, ${ }^{25}$ accelerating polyethylene wear and producing osteolysis, ${ }^{26}$ could not be confirmed in this series, since focal osteolysis around the malpositioned tibial component was only observed in four $(18.2 \%)$ of 22 ankles in the control group and none in the hindfoot fusion group. 
Table VIII. Details of revised cases

\begin{tabular}{|c|c|c|c|c|c|c|}
\hline Gender & $\begin{array}{l}\text { Age } \\
\text { (yrs) }\end{array}$ & Group & $\begin{array}{l}\text { Months to } \\
\text { failure }\end{array}$ & Reason for failure & Primary revision & Prognosis \\
\hline $\mathrm{F}$ & 77 & Hindfoot fusion & 11 & Aseptic loosening of the tibial component & Revision of tibial component & Good \\
\hline M & 68 & Control & 15 & Aseptic loosening of the tibial component & Revision of tibial component & Good \\
\hline $\mathrm{F}$ & 67 & Control & 18 & Aseptic loosening of the tibial component & Arthrodesis & Good \\
\hline $\mathrm{F}$ & 64 & Hindfoot fusion & 22 & Aseptic loosening of the tibial component & Arthrodesis & Good \\
\hline M & 51 & Control & 24 & Painful arthrofibrosis & Arthrodesis & Good \\
\hline $\mathrm{F}$ & 59 & Control & 26 & $\begin{array}{l}\text { Recurrent polyethylene component } \\
\text { dislocation }\end{array}$ & Arthrodesis & Good \\
\hline M & 72 & Control & 26 & Aseptic loosening of the tibial component & Revision of tibial component & Good \\
\hline M & 59 & Control & 27 & Aseptic loosening of the tibial component & Revision of tibial component & $\begin{array}{l}\text { Supramalleolar osteotomy } \\
\text { due to valgus malalignment }\end{array}$ \\
\hline M & 25 & Control & 50 & $\begin{array}{l}\text { Aseptic loosening of the tibial and talar } \\
\text { components }\end{array}$ & $\begin{array}{l}\text { Revision of tibial and talar } \\
\text { components }\end{array}$ & $\begin{array}{l}\text { Converted to arthrodesis due } \\
\text { to recurrent loosening }\end{array}$ \\
\hline $\mathrm{F}$ & 48 & Control & 58 & Painful arthrofibrosis & Arthrodesis & Good \\
\hline
\end{tabular}

We believe that several factors contributed to the finding that the outcome for replaced ankles in the hindfoot fusion group was not inferior to that for the control group. One is the role of the mobile bearing. The function of the subtalar joint in mechanically coupling the foot and the tibia involves transformation of rotation into calcaneal eversioninversion and vice versa. ${ }^{27}$ Interestingly, this transfer of movement does not substantially decrease after subtalar fusion as the ankle joint provides a significant part of this transfer of movement. ${ }^{28}$ In a replaced ankle, the flat surfaces between the polyethylene bearing and the tibial component allow a certain degree of rotation. We speculate that this plays an important role in the transfer of movement between the calcaneum and the tibia in ankles with a fused hindfoot.

Another factor is the compensatory increase in ROM at the knee. The loss of the dynamic function of the subtalar and transverse tarsal joints results in a rigid mid- and hindfoot and reduces the ROM of the ankle. ${ }^{29,30}$ However, gait analysis has revealed that reduced movement of the ankle is compensated by increased flexion of the knee and greater knee extension moments..$^{29,30}$ Fixation of the tibial component independently of the fusion of the syndesmosis can also contribute to the low failure rate by minimising the effects of hindfoot fusion. Hindfoot fusion increases the displacement across the anterior tibiofibular ligament, and can cause failure of designs that rely on syndesmosis fusion such as the Agility system (DePuy, Warsaw, Indiana). ${ }^{31}$ Therefore, preserving the normal anatomy of the syndesmosis can be important if TAR is combined with hindfoot fusion.

We had conflicting results concerning the timing of any hindfoot fusion on the outcome of TAR. Soft-tissue related or secondary problems, including scarring and bony impingement, tended to develop more frequently with twostaged fusion $(p=0.054)$, whereas instability or dislocation of the polyethylene were observed only in the simultaneous fusion group. Concerning the extent of fusion, isolated talonavicular fusion was not performed despite its effec- tiveness in the correction of deformity ${ }^{32}$ either because most of our cases with endstage osteoarthritis of the ankle had associated adjacent arthritis primarily involving the subtalar joint, or because they had deformities requiring isolated subtalar or combined subtalar and talonavicular fusion. ${ }^{33}$ Fusion of the calcaneocuboid joint was added only when there was arthritis of this joint because sparing the joint has advantages in reducing operative time, risk of nonunion, ${ }^{15,34}$ and further adjacent joint arthritis. ${ }^{33,35}$ TAR with isolated subtalar fusion and double or triple fusion showed similar results. This can be related to the similar gait patterns after isolated subtalar $v$ s triple fusion as fusion of the subtalar joint also decreases the movement of the talonavicular and calcaneocuboid joints. ${ }^{35,36}$ We were also concerned about the possible development of osteonecrosis of the talus and subsequent loosening of the talar component in the hindfoot fusion group due to aggressive denuding and devascularisation up to the sinus tarsi during subtalar fusion. Fortunately, osteonecrosis of the talus did not occur, and the one case $(0.3 \%)$ of loosening of the talar component was in the control group.

It was interesting to note in the hindfoot fusion group that the hindfoot complex was anteriorly displaced immediately after surgery, but was relocated to the mortise at the last follow-up. This change in the contact point ratio $<40$ can be explained by a small amount of shortening that occurs in the process of fusion. The relocation of the talar component is mainly due to the realignment of the ligaments that help to restore the normal axis of rotation as described by Wood et al. ${ }^{6}$ Furthermore, this restoration in the sagittal plane is blocked when the tibial component is placed facing anteriorly. Therefore, the surgeon should take care not to increase the posterior slope, especially in patients undergoing subtalar fusion.

Our results should be interpreted with caution. Firstly, there were some differences in the demographics of the two groups with the mean height and weight being lower in the hindfoot fusion group, as there were more women in this group compared with the control group $(\mathrm{p}=0.015)$. How- 
ever, the body mass index was similar between the groups. Furthermore the different gender distribution seemed to be related to the aetiology of the arthritis, with the proportion of rheumatoid arthritis being higher in the hindfoot fusion group, and we believe that this is partially related to the characteristics of rheumatoid arthritis involving multiple joints. Since the results of TAR in rheumatoid patients are reported to be $\operatorname{good}^{37}$ or not inferior to those in patients with osteoarthritis, ${ }^{38}$ we assumed that the different distribution of aetiology would not seriously compromise our findings. Another important limitation is that the preoperative status of the two groups was different. Thus, comparing the outcomes of the two groups could be questioned. However, since it is apparent that ankles requiring simultaneous hindfoot fusion had more complex problems and worse pre-operative function, improvement to a level equivalent to the control group is meaningful. Furthermore, the modified AOFAS score ${ }^{19}$ has been used to account for the loss of movement in the subtalar joint but we accept this has not been validated and may not be ideal for the assessment of the ankle and fused hindfoot complex.

In conclusion, hindfoot fusion is feasible, even simultaneously with TAR, and has minimal adverse effect on TAR up to midterm follow-up. The clinical outcomes of TAR with hindfoot fusion are comparable to those of TAR alone. Thus, hindfoot fusion should be performed in conjunction with TAR when indicated.

\section{Listen live}

Listen to the abstract of this article at www.jbjs.org.uk/interactive/audio

The authors wish to thank D. S. Jang for graphical assistance.

No benefits in any form have been received or will be received from a commercial party related directly or indirectly to the subject of this article.

\section{References}

1. Doets HC, Brand R, Nelissen RG. Total ankle arthroplasty in inflammatory joint dis ease with use of two mobile-bearing designs. J Bone Joint Surg [Am] 2006;88A:1272-84.

2. Haskell A, Mann RA. Ankle arthroplasty with preoperative coronal plane deformity: short-term results. Clin Orthop 2004;424:98-103.

3. Hobson SA, Karantana A, Dhar S. Total ankle replacement in patients with signif icant pre-operative deformity of the hindfoot. J Bone Joint Surg [Br]2009;91-B:481-6.

4. Kim BS, Choi WJ, Kim YS, Lee JW. Total ankle replacement in moderate to severe varus deformity of the ankle. J Bone Joint Surg [Br] 2009;91-B:1183-90.

5. Wood PL, Deakin S. Total ankle replacement: the results in 200 ankles. J Bone Joint Surg [Br] 2003;85-B:334-41.

6. Wood PL, Prem H, Sutton C. Total ankle replacement: medium-term results in 200 Scandinavian total ankle replacement. J Bone Joint Surg [Br] 2008;90-B:605-9.

7. Wood PL, Sutton C, Mishra V, Suneja R. A randomised, controlled trial of two mobile-bearing total ankle replacements. J Bone Joint Surg [Br] 2009;91-B:69-74.

8. Conti SF, Wong YS. Complications of total ankle replacement. Clin Orthop 2001;391:105-14.

9. Gould JS, Alvine FG, Mann RA, Sanders RW, Walling AK. Total ankle replacement: a surgical discussion. Part I: replacement systems, indications, and contraindications. Am J Orthop 2000;29:604-9.

10. Hintermann B. Total ankle arthroplasty: historical overview, current concepts, and future perspectives. New York: Springer Wein, 2005
11. Hintermann B, Valderrabano V. Total ankle replacement. Foot Ankle Clin 2003;8:375-405

12. Spirt AA, Assal M, Hansen ST Jr. Complications and failure after total ankle arthroplasty. J Bone Joint Surg [Am] 2004;86-A:1172-8.

13. Burton DC, OIney BW, Horton GA. Late results of subtalar distraction fusion. Foot Ankle Int 1998;19:197-202.

14. de Heus JA, Marti RK, Besselaar PP, Albers GH. The influence of subtalar and triple arthrodesis on the tibiotalar joint: a long-term follow-up study. J Bone Joint Surg [Br] 1997;79-B:644-7.

15. Graves SC, Mann RA, Graves KO. Triple arthrodesis in older adults: results after long-term follow-up. J Bone Joint Surg [Am] 1993;75-A:355-62.

16. Mangone PG, Fleming LL, Fleming SS, et al. Treatment of acquired adult planovalgus deformities with subtalar fusion. Clin Orthop 1997;341:106-12.

17. Mann RA, Beaman DN, Horton GA. Isolated subtalar arthrodesis. Foot Ankle Int 1998;19:511-19

18. Rammelt S, Grass R, Zawadski T, Biewener A, Zwipp H. Foot function after subtalar distraction bone-block arthrodesis: a prospective study. J Bone Joint Surg [Br] 2004;86-B:659-68.

19. Easley ME, Trnka HJ, Schon LC, Myerson MS. Isolated subtalar arthrodesis. J Bone Joint Surg [Am] 2000;82-A:613-24.

20. Marti RK, de Heus JA, Roolker W, Poolman RW, Besselaar PP. Subtalar arthrodesis with correction of deformity after fractures of the os calcis. J Bone Joint Surg [Br] 1999;81-B:611-16.

21. Southwell RB, Sherman FC. Triple arthrodesis: a long-term study with force plate analysis. Foot Ankle 1981;2:15-24

22. Hintermann B, Vanderrabano V, Dereymaeker G, Dick W. The HINTEGRA ankle: rationale and short-term results of 122 consecutive ankles. Clin Orthop 2004;424:57-

23. Kitaoka HB, Alexander IJ, Adelaar RS, et al. Clinical rating systems for the anklehindfoot, midfoot, hallux, and lesser toes. Foot Ankle Int 1994;15:349-53.

24. Takakura Y, Tanaka Y, Kumai T, Tamai S. Low tibial osteotomy for osteoarthritis of the ankle: results of a new operation in 18 patients. J Bone Joint Surg [Br] 1995;77B:50-4

25. Stamatis ED, Myerson MS. How to avoid specific complications of total ankle replacement. Foot Ankle Clin 2002;7:765-89.

26. Kadoya Y, Kobayashi A, Ohashi H. Wear and osteolysis in total joint replacements. Acta Orthop Scand Supp/ 1998;278:1-16.

27. Close JR, Inman VT, Poor PM, Todd FN. The function of the subtalar joint. Clin Orthop 1967;50:159-79.

28. Hintermann B, Nigg BM. Influence of arthrodeses on kinematics of the axially loaded ankle complex during dorsiflexion/plantarflexion. Foot Ankle Int 1995;16:633-

29. Beischer AD, Brodsky JW, Pollo FE, Peereboom J. Functional outcome and gait analysis after triple or double arthrodesis. Foot Ankle Int 1999;20:545-53.

30. Wu WL, Huang PJ, Lin CJ, et al. Lower extremity kinematics and kinetics during level walking and stair climbing in subjects with triple arthrodesis or subtalar fusion. Gait Posture 2005;21:263-70.

31. Witkowski GP, Bilkhu SK, Siskosky MJ, et al. Effects of hindfoot constraint on syndesmotic displacement. Foot Ankle Int 2009;30:367-74.

32. O'Malley MJ, Deland JT, Lee KT. Selective hindfoot arthrodesis for the treatment of adult acquired flatfoot deformity: an in vitro study. Foot Ankle Int 1995;16:411-17.

33. Knupp M, Schuh R, Stufkens SA, Bolliger L, Hintermann B. Subtalar and talonavicular arthrodesis through a single medial approach for the correction of severe planovalgus deformity. J Bone Joint Surg [Br] 2009;91-B:612-15.

34. Sammarco VJ, Magur EG, Sammarco GJ, Bagwe MR. Arthrodesis of the subtalar and talonavicular joints for correction of symptomatic hindfoot malalignment. Foot Ankle Int 2006;27:661-6.

35. Astion DJ, Deland JT, Otis JC, Kenneally S. Motion of the hindfoot after simulated arthrodesis. J Bone Joint Surg [Am] 1997;79-A:241-6.

36. Chen CH, Huang PJ, Chen TB, et al. Isolated talonavicular arthrodesis for talonavicular arthritis. Foot Ankle Int 2001;22:633-6.

37. van der Heide HJ, Schutte B, Louwerens JW, van den Hoogen FH, de Waal Malefijt MC. Total ankle prostheses in rheumatoid arthropathy: outcome in 52 patients followed for 1-9 years. Acta Orthop 2009:80:440-4

38. Kofoed H, Sorensen TS. Ankle arthroplasty for rheumatoid arthritis and osteoarthritis: prospective long-term study of cemented replacements. J Bone Joint Surg [Br] 1998;80-B:328-32. 